02

\title{
Исследование плазмонно-связанной флуоресценции родамина $6 Ж$ на тонких никелевых пленках
}

\author{
() А.Д. Дмитриев, А.М. Салецкий \\ Московский государственный университет им. М.В. Ломоносова, физический фракультет, \\ 119991 Москва, Россия \\ ฯ e-mail: sam@physics.msu.ru \\ Поступила в редакцию 19.02.2019 г. \\ В окончательной редакции 19.02.2019 г. \\ Принята к публикации 09.04.2019 г.
}

\begin{abstract}
Экспериментально и теоретически исследована плазмонно-связанная люминесценция молекул красителя родамина 6Ж на тонкой никелевой пленке. Пространственное распределение флуоресценции молекул красителя, рассчитанное с использованием метода матриц переноса для описания плоскослоистых сред, хорошо согласуется с полученными экспериментальными диаграммами пространственного распределения излучения разной поляризации. Экспериментально установлена эффективность связи люминофора с плазмонами никелевой пленки.
\end{abstract}

Ключевые слова: плазмоны-поляритоны, поверхностная флуоресценция, тонкие пленки.

DOI: $10.21883 /$ OS.2019.08.48039.68-19

\section{Введение}

В последние годы интенсивно проводятся научные исследования, направленные на модификацию и управление оптическими характеристиками органических люминесцирующих материалов и композитов на их основе, a также на усиление излучательной способности, в том числе и с использованием плазмонного резонанса металлических наноструктур [1-3]. Эта так называемая плазмонно-связанная флуоресценция (SPCE) обусловлена взаимодействием поверхностных плазмонов металла и возбужденного состояния флуорофора. Размещая излучатели вблизи металлических наноструктур, можно добиться значительного усиления люминесценции из-за эффекта плазмонного резонанса (SPR). Металлические наноструктуры, поддерживающие моды локализованных и распространяющихся плазмонов, могут приводить к заметному усилению локальных электромагнитных полей и обусловливают сильно модифицированную локальную плотность оптических состояний [4,5]. Плазмонно-связанная флуоресценция открывает путь для многочисленных применений во многих областях биомедицины, значительный прогресс при этом наблюдается при создании биологических сенсоров [6,7]. Обычно такие наноструктуры изготовляются из благородных металлов (золото и серебро).

В недавние времена сочетание плазмоники с магнетизмом стало активной темой исследований для достижения новых функций в наносистемах [8,9]. Однако для таких целей не пригодны благородные металлы, которые требуют очень высокие значения магнитных полей для проявления магнитооптической активности. С другой стороны, ферромагнитный металл обладает большой магнитооптической активностью и также подходит для плазмоники. В связи с этим ферромагнитные металлы, такие как кобальт $(\mathrm{Co})$ и никель $(\mathrm{Ni})$, привлекли особое внимание исследователей из-за их магнитооптических свойств. Никель является химически инертным металлом, и поэтому его использование в сенсоре SPR является предпочтительным.

В настоящей работе теоретически и экспериментально исследована поверхностная плазмонно-связанная флуоресценция молекул родамина 6Ж (Р6Ж) на никелевых пленках.

\section{Материалы и методы}

\section{Приготовление образцов}

На предметное стекло $26 \times 30 \times 1 \mathrm{~mm}$ напылялся слой никеля толщиной $13 \mathrm{~nm}$, а затем слой $\mathrm{SiO}_{2}$ толщиной $10 \mathrm{~nm}$. Толщина металлической пленки была выбрана на основе оптимизации кривых отражения, полученных методом матриц переноса. Критерием отбора служила глубина минимума поверхностного плазмонного резонанса при варьировании толщины слоя металла. Слой $\mathrm{SiO}_{2}$ служит разделителем между поверхностью металла и молекулами люминофора, его наличие препятствует быстрому тушению красителя, которое является доминирующим процессом вблизи металла $(\leq 10 \mathrm{~nm})$.

Размещение молекул люминофора на подложке осуществлялось методом центрифугирования. Для этого был приготовлен однопроцентный раствор поливинилового спирта (PVA) в воде, содержащей краситель Р6Ж с молярной концентрацией $1 \cdot 10^{-3} \mathrm{~mol} / \mathrm{l}$. Нанесение производилось на спин-коутере G3P-8 (Specialty Coating 


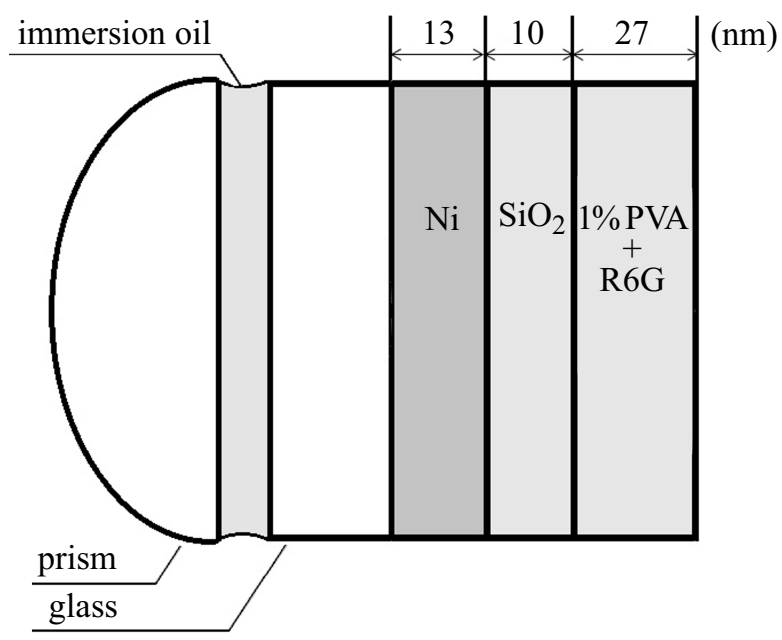

Рис. 1. Структура образца для SPCE.

Systems) в двухступенчатом скоростном режиме - $8 \mathrm{~s}$ на скорости 800 rpm, 20 s на скорости $2400 \mathrm{rpm}$.

Измерение толщины получившейся полимерной пленки производилось на сканирующем зондовом микроскопе (C3M) SMENA на платформе Solver P47H-PRO компании NT-MDT. Для этого были подготовлены образцы, представляющие собой пленки PVA с Р6Ж, нанесенные на предметные стекла. Так как условия нанесения для референтных и исследуемых образцов оставались неизменными, можно оценить толщину полимерной пленки экспериментальных образцов $\sim 30 \mathrm{~nm}$. Покрытые стекла были прикреплены к полуцилиндрической призме из стекла ВК7 через иммерсионную жидкость. Структура такого комбинированного образца показана на рис. 1.

\section{Спектрально-флуоресцентные измерения}

Комбинированный образец был расположен на гониометре ГС-30, что позволяло возбуждать образец и наблюдать излучение под любым желаемым углом относительно вертикальной оси. Образец можно освещать через призму (конфигурация Кречмана). Также образец может возбуждаться со стороны воздуха. Это называется обратной конфигурацией Кречмана. Для наблюдения SPCE применялась обратная конфигурация Кречмана, т.е. источник возбуждения (лазер $532 \mathrm{~nm}$ ) находился с обратной стороны (по отношению к призме). Регистрация излучения проводилась через поляроид (для выделения заданной поляризации) с помощью световода, выход которого был подключен к спектрофлуориметру Солар СМ2203.

\section{Теория}

Для получения картины пространственного распределения излучения, высвеченного молекулой красителя, находящейся в слоистой среде, воспользуемся подходом, предложенным в [10]. Будем рассматривать молекулу люминофора как осциллирующий диполь, поле которого можно записать в виде

$$
\begin{gathered}
\mathbf{E}=-\frac{1}{4 \pi \varepsilon} \nabla \times\left[\nabla \times \mathbf{p} \frac{\exp (i k r)}{r}\right], \\
\mathbf{H}=\frac{i k c}{4 \pi} \nabla \times \mathbf{p} \frac{\exp (i k r)}{r},
\end{gathered}
$$

где $\mathbf{p}-$ дипольный момент, $k-$ волновое число, $\varepsilon-$ диэлектрическая проницаемость среды, $r$ - модуль радиуса-вектора, $c$ - скорость света. Далее применим двумерное преобразование Фурье к сферической волне $\exp (i k r) / r$ в (1) и получим излучение диполя как сумму плоских волн двух типов: эванесцентных и распространяющихся в дальнюю зону. Так как после преобразования диполь будет представлен как источник плоских волн, можно записать поле в каждом слое как сумму двух плоских волн, бегущих в противоположных направлениях. Для описания плоскослоистых сред применим метод матриц переноса [11]. Слоистую среду, содержащую $N$ зон, из которых первая и последняя являются бесконечными полупространствами, а остальные $N-2$ это слои образца с диэлектрическими $\varepsilon_{m}$ и магнитными $\mu_{m}$ проницаемостями и толщинами $h_{m}=z_{m}-z_{m-1}$, где $z_{m}$ положение соответствующей границы раздела, можно охарактеризовать матрицами

$$
\begin{gathered}
\mathbf{S}_{m}^{P}=\frac{1}{2}\left(\begin{array}{cc}
\frac{n_{m}}{n_{m+1}}+\frac{\cos \left(\theta_{m}\right)}{\cos \left(\theta_{m+1}\right)} & \frac{n_{m}}{n_{m+1}}-\frac{\cos \left(\theta_{m}\right)}{\cos \left(\theta_{m+1}\right)} \\
\frac{n_{m}}{n_{m+1}}-\frac{\cos \left(\theta_{m}\right)}{\cos \left(\theta_{m+1}\right)} & \frac{n_{m}}{n_{m+1}}+\frac{\cos \left(\theta_{m}\right)}{\cos \left(\theta_{m+1}\right)}
\end{array}\right), \\
\mathbf{S}_{m}^{S}=\frac{1}{2}\left(\begin{array}{cc}
1+\frac{n_{m} \cos \left(\theta_{m}\right)}{n_{m+1} \cos \left(\theta_{m+1}\right)} & 1-\frac{n_{m} \cos \left(\theta_{m}\right)}{n_{m+1} \cos \left(\theta_{m+1}\right)} \\
1-\frac{n_{m} \cos \left(\theta_{m}\right)}{n_{m+1} \cos \left(\theta_{m+1}\right)} & 1+\frac{n_{m} \cos \left(\theta_{m}\right)}{n_{m+1} \cos \left(\theta_{m+1}\right)}
\end{array}\right), \\
\mathbf{P}_{m}=\left(\begin{array}{cc}
\exp \left(i k_{m} h_{m} \cos \left(\theta_{m}\right)\right) & 0 \\
0 & \exp \left(-i k_{m} h_{m} \cos \left(\theta_{m}\right)\right)
\end{array}\right),
\end{gathered}
$$

где $n_{m}-$ показатель преломления, $k_{m}-$ волновое число и $\theta_{m}-$ полярный угол $m$-го слоя. Матрицы $\mathbf{S}_{m}^{(P, S)}$ отвечают за согласование полей на границах раздела слоев $m$ и $m+1$, индексы $(P, S)$ означают поляризацию волн, а матрицы $\mathbf{P}_{m}$ - за распространение волн внутри каждого $m$-го слоя, так что связь между полями в соседних слоях запишется в виде $\mathbf{a}_{m+1}=S_{m} P_{m} \mathbf{a}_{m}$, где $\mathbf{a}_{m}$ - вектор-столбец с коэффициентами разложения электрического поля в слое $m$.

Используя граничные условия и решая систему уравнений для коэффициентов разложения, можно получить выражение для интересующей нас величины интенсивности электромагнитного поля как функции 
направления в пространстве:

$$
\begin{aligned}
& \mathbf{I}\left(\theta_{N}\right)=\frac{3 n_{N}^{3} \mathbf{r}}{8 \pi n_{M}^{3}|\mathbf{p}|^{2}} \operatorname{Re}\left(\frac { \operatorname { c o s } ^ { 2 } ( \theta _ { N } ) } { | \operatorname { c o s } ( \theta _ { M } ) | ^ { 2 } } \left[\left(a_{\perp N}^{P} \mathbf{z} \cdot \mathbf{p}+a_{\| N}^{P} \boldsymbol{\eta} \cdot \mathbf{p}\right)\right.\right. \\
& \left.\left.\times\left(a_{\perp N}^{P} \mathbf{z} \cdot \mathbf{p}+a_{\| N}^{P} \boldsymbol{\eta} \cdot \mathbf{p}\right)^{*}+a_{\| N}^{S} \boldsymbol{\varphi} \cdot \mathbf{p}\left(a_{\| N}^{S} \boldsymbol{\varphi} \cdot \mathbf{p}\right)^{*}\right]\right),
\end{aligned}
$$

где $n_{M}$ и $n_{N}$ - показатели преломления среды, в которой находится диполь, и среды, в которой необходимо найти распределение излучения, $\mathbf{r}$ - радиус-вектор, $(\mathbf{z}, \boldsymbol{\eta}, \boldsymbol{\varphi})$ - единичные векторы волнового вектора $\mathbf{k}$ в цилиндрической системе координат. Коэффициенты поля $a_{\perp N}^{P}, a_{\| N}^{P}$ и $a_{\| N}^{S}$, в которых индексы $(\perp, \|)$ означают дипольную ориентацию, $N$ - номер слоя (в данном случае это последний полубесконечный слой), можно найти из следующего уравнения

$$
\begin{aligned}
\left(\begin{array}{c}
a_{(\perp, \|) N}^{(P, S)} \\
a_{(\perp, \|) l}^{(P, S)^{*}}
\end{array}\right) & =\left(\left(\begin{array}{ll}
1 & 0 \\
0 & 0
\end{array}\right)-\mathbf{A}_{N, 1}\left(\begin{array}{ll}
0 & 0 \\
0 & 1
\end{array}\right)\right)^{-1} \\
& \times \mathbf{A}_{N, M+1} \cdot \mathbf{q}_{\perp, \|}^{(P, S)}
\end{aligned}
$$

где

$$
\begin{gathered}
\mathbf{q}_{\perp}^{P}=\left(\begin{array}{c}
1 \\
-1
\end{array}\right) \sin (\theta)_{M}, \\
\mathbf{q}_{\|}^{P}=-\left(\begin{array}{l}
1 \\
1
\end{array}\right) \cos \left(\theta_{M}\right), \quad \mathbf{q}_{\|}^{S}=\left(\begin{array}{c}
-1 \\
1
\end{array}\right)
\end{gathered}
$$

в зависимости от ориентации и поляризации,

$$
\begin{aligned}
\mathbf{A}_{N, 1} & =\mathbf{P}_{N} \mathbf{S}_{N, N-1} \ldots \mathbf{P}_{2} \mathbf{S}_{21} \mathbf{P}_{1}, \\
\mathbf{A}_{N, M+1} & =\mathbf{P}_{N} \mathbf{S}_{N, N-1} \mathbf{P}_{N-1} \ldots \mathbf{P}_{M+1} .
\end{aligned}
$$

От матриц (2), связывающих коэффициенты разложения электрического поля в разных слоях, можно перейти к матрицам, связывающим тангенциальные компоненты электрического и магнитного полей:

$$
\mathbf{M}_{m}=\left(\begin{array}{cc}
\cos \left(\beta_{m}\right) & \frac{-i}{p_{m}} \sin \left(\beta_{m}\right) \\
-i p_{m} \sin \left(\beta_{m}\right) & \cos \left(\beta_{m}\right)
\end{array}\right),
$$

где $p_{m}=\left(\varepsilon_{m} / \mu_{m}\right)^{1 / 2} \cos \left(\theta_{m}\right)$ в случае $S$-поляризации и $p_{m}=\left(\mu_{m} / \varepsilon_{m}\right)^{1 / 2} \cos \left(\theta_{m}\right)$ в случае $P$-поляризации, $\beta_{m}=2 \pi / \lambda n_{m} \cos \left(\theta_{m}\right) h_{m}, \lambda$ - длина волны света в вакууме.

Таким образом, матрицы (7) устанавливают соотношения $\mathbf{Q}_{m-1}(z)=\mathbf{M}_{m} \mathbf{Q}_{m}(z)$, где $\mathbf{Q}_{m}(z)-$ вектор-столбец, содержащий компоненты электромагнитного поля в $m$-м слое $\left(E_{y m}(z), H_{x m}(z)\right)$ для $S$-поляризации и $\left(H_{y m}(z)\right.$, $\left.E_{x m}(z)\right)$ для $P$-поляризации. Зная матрицы (7) для каждого слоя, можно найти характеристическую матрицу структуры: $\mathbf{M}=\prod_{m=2}^{N-1} \mathbf{M}_{m}$, элементы которой определяют амплитудные коэффициенты отражения и пропускания:

$$
\begin{aligned}
& r=\frac{\left(M_{11}+M_{12} p_{N}\right) p_{1}-\left(M_{21}+M_{22} p_{N}\right)}{\left(M_{11}+M_{12} p_{N}\right) p_{1}+\left(M_{21}+M_{22} p_{N}\right)}, \\
& t=\frac{2 p_{1}}{\left(M_{11}+M_{12} p_{N}\right) p_{1}+\left(M_{21}+M_{22} p_{N}\right)},
\end{aligned}
$$

где $M_{a \beta}$ - матричный элемент характеристической матрицы М. Формулы (8) позволяют определить коэффициент отражения $R=|r|^{2}$ и используются для нахождения электромагнитного поля в произвольной точке $k$-го слоя:

$$
\begin{gathered}
H_{y k}(z, x)=H_{y k}(z) \exp \left(i \frac{2 \pi}{\lambda} n_{1} \sin \left(\theta_{1}\right) x\right), \\
E_{x k}(z, x)=E_{x k}(z) \exp \left(i \frac{2 \pi}{\lambda} n_{1} \sin \left(\theta_{1}\right) x\right), \\
E_{z k}(z, x)=\frac{n_{1} \sin \left(\theta_{1}\right)}{\varepsilon_{k}} H_{y k}(z) \exp \left(i \frac{2 \pi}{\lambda} n_{1} \sin \left(\theta_{1}\right) x\right),
\end{gathered}
$$

где $H_{y k}(z, x), E_{x k}(z, x), E_{z k}(z, x)$ - составляющие электромагнитного поля $P$-поляризации, величины $H_{y k}(z)$ и $E_{x k}(z)$ находятся из соотношения

$$
\left(\begin{array}{c}
H_{y k}(z) \\
E_{x k}(z)
\end{array}\right)=\mathbf{N}_{k}(z) \prod_{m=k}^{N-1} \mathbf{M}_{m} \mathbf{Q}_{N-1},
$$

где $\mathbf{N}_{k}(z)$ - матрица, обратная $\mathbf{M}_{\mathbf{k}}$ с $h_{m}=z-z_{m-1}$, а $\mathbf{Q}_{N-1}=\left(\begin{array}{c}t \\ -p_{N} t\end{array}\right) n_{1}$. Аналогично можно получить выражения для компонент поля $S$-поляризации.

Резонансное поведение коэффициентов отражения и преломления поля на поверхности металла - основные следствия процесса поверхностного плазмонного резонанса, и так как матричный метод позволяет рассчитать данные величины, он хорошо подходит для оценки процессов плазмонного поглощения в плоскослоистых структурах, содержащих гладкие металлические пленки, слой разделителя и слой PVA - как в нашем случае.

\section{Результаты и их обсуждение}

На рис. 2 представлены теоретические кривые диаграммы направленности излучения (производилось усреднение по положению диполя в слое PVA) и полученные экспериментальные данные. Кривые 1 и 2 были рассчитаны по формуле (3), в которой использовались коэффициенты поля соответствующих поляризаций. В слоистой среде воздух со стороны пленки PVA считался первой средой, пленка PVA с красителем второй и так далее до $N$-й среды - стеклянной призмы. Для расчета свободного излучения (кривая 2) последней средой в структуре считалась среда с номером 1 (т.е. в воздух).

Кривая 1 на рис. 2 описывает $P$-поляризованное плазмонно-связанное излучение, наблюдаемое со стороны 


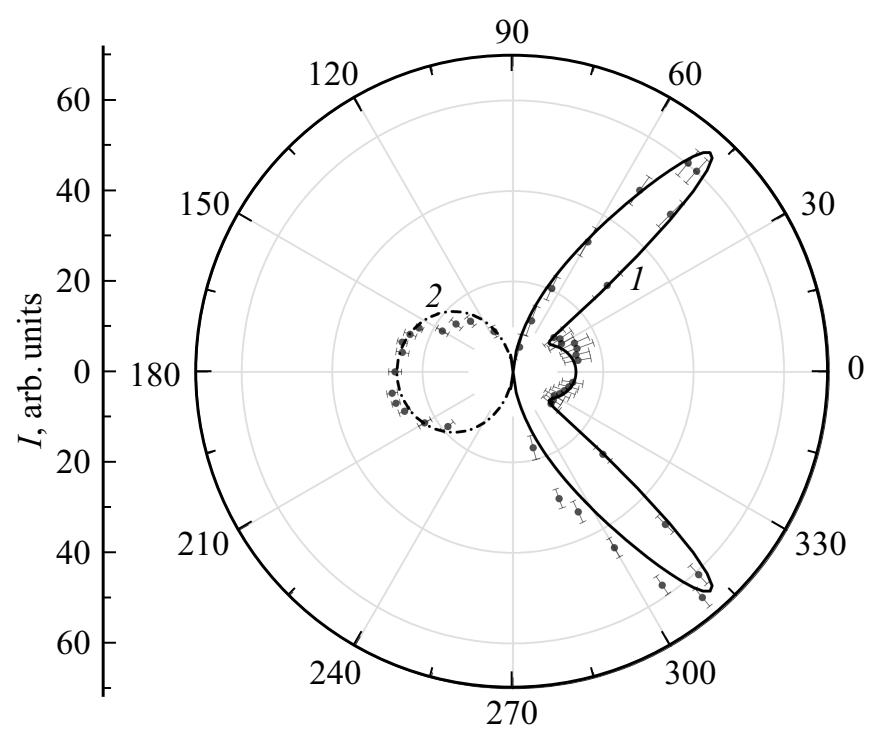

Рис. 2. Теоретически рассчитанные диаграммы направленности $P$-поляризованной люминесценции $(1)$ и несвязанного излучения (2) (суммарно $P$ - и $S$-поляризаций); точки экспериментальные значения интенсивности люминесценции родамина $6 Ж(\lambda=565 \mathrm{~nm})$.

призмы. Максимальные значения интенсивности наблюдается при $50.0^{\circ}$ и $48.7^{\circ}$ относительно оси симметрии системы. Ширина пика на полувысоте достигает $\approx 18^{\circ}$, что объясняется большой мнимой частью диэлектрической проницаемости никеля $\varepsilon=-7.87+i 12.01$. В то же время с обратной стороны образца, наблюдается излучение не связанных с подложкой диполей (кривая 2). Это свободное излучение не сопровождается ближнепольным взаимодействием излучателей и поверхностных плазмон-поляритонов, для него характерно отсутствие какого-либо выделенного направления и состояния поляризации, поэтому на рис. 2 свободное излучение включает в себя сумму обеих составляющих поляризации. Чем дальше диполь находится от металлической пленки, тем слабее его взаимодействие с поверхностными плазмон-поляритонами и тем больше доля свободного излучения (кривая 2) по сравнению со связанным (кривая 1). Экспериментальные исследования (точки на рис. 2) показали хорошее согласие с теоретическими расчетами. Свойство высокой направленности связанного излучения берет начало в резонансной природе поверхностного плазмонного резонанса, реализующегося для узкого диапазона углов или длин волн. И так как связанное излучение является обратным процессом по отношению к плазмонному резонансу, то условия получения резонанса ограничивают и возможные направления поверхностного плазмонно-связанного излучения в пространстве. В пиковых значениях это излучение в 2.5 раза превышает излучение несвязанных диполей.

На рис. 3 представлена теоретически рассчитанная (кривая 1) и измеренная (точки) $S$-поляризованная лю-

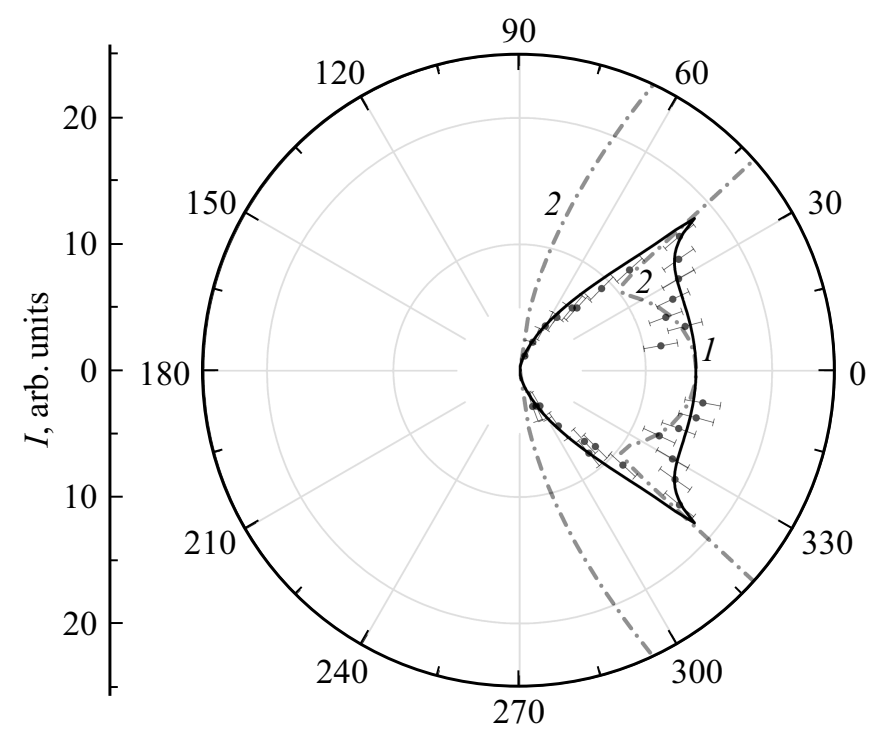

Рис. 3. Теоретически рассчитанные диаграммы направленности $S$-поляризованной люминесценции $(1)$ и $P$-поляризованного излучения (2). Точки - экспериментальные значения интенсивности люминесценции Р6Ж $(\lambda=565 \mathrm{~nm})$. Все величины соответствуют излучению „в призму“.

минесценция Р6Ж, наблюдавшаяся „В призму“. Так как поверхностные плазмон-поляритоны являются $P$-поляризованными волнами, то следует ожидать, что связанное излучение будет иметь преимущественную $P$-поляризованную составляющую. На практике наряду с доминирующей $P$-компонентой наблюдается более слабая $S$-составляющая излучения. Для сравнения двух поляризаций свечения, наблюдавшегося „в призму“, на рис. 3 приведена в масштабе теоретическая кривая для Р- излучения (кривая 2). Видно, что $S$-излучение „в призму“ не обладает свойством ярко выраженной направленности и значительно слабее $P$-излучения.

Из представленных экспериментальных данных можно получить оценку эффективности связи люминофора с никелевой подложкой:

$$
\eta=\frac{I_{P}}{I_{P}+I_{S}+I_{P r}}=45.6 \%,
$$

где $I_{P}, I_{S}, I_{P r}-$ интегральные интенсивности $P$-, $S$-излучения „в призму“ и свободного излучения с обратной стороны соответственно. Величина (11) показывает долю молекул Р6Ж в образце, излучение которых наблюдается как поверхностное плазмонно-связанное излучение.

На рис. 4 представлена зависимость коэффициента отражения образца от угла падения (наблюдения) (кривая 1) при падении света со стороны призмы, рассчитанная методом матриц переноса (используется первая из формул (8)), для $\lambda=565 \mathrm{~nm}$. Из рис. 4 видно, что резкое увеличение коэффициента отражения наблюдается при угле полного внутреннего отражения $\left(\sim 41.5^{\circ}\right)$. При дальнейшем увеличении угла падения для зависимости 


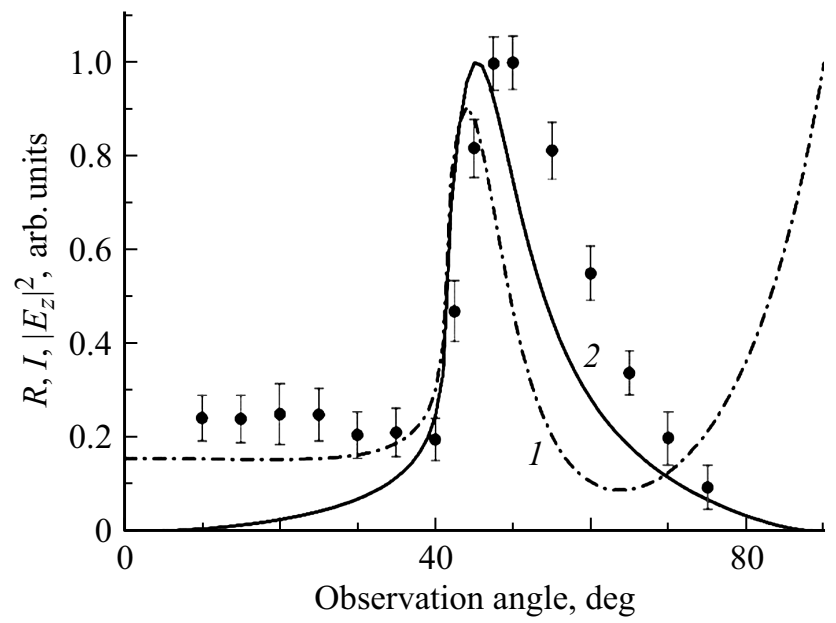

Рис. 4. Теоретически рассчитанные коэффициент отражения для $P$-поляризованного света $(1)$ и средний квадрат $z$-проекции поля в слое ПВС, деленный на средний квадрат $z$-проекции поля в первой среде (2). Поле (2) создается падающей со стороны призмы $P$-поляризованной волной, которая рождает плазмон-поляритоны на границе металл/PVA и рассчитывается в середине слоя PVA. Точки - экспериментальные значения интенсивности люминесценции Р6Ж $(\lambda=565 \mathrm{~nm})$. Все величины нормированы на их максимальные значения.

$R(\theta)$ наблюдается широкий минимум отражения. Такое поведение $R(\theta)$ можно объяснить большой величиной мнимой части диэлектрической проницаемости, ответственной за ширину провала. В такой ситуации определение истинного положения плазмонного резонанса по положению минимума отражения весьма затруднительно.

Как видно из рис. 4, зарегистрированная интенсивность связанной люминесценции (точки на рис. 4) имеет максимальное значение при $\theta \approx 50^{\circ}$, в то время как минимум отражения наблюдается при $\theta \approx 63^{\circ}$. Наблюдаемое различие можно объяснить сильным поглощением никеля и сделать вывод, что в данном случае минимум отражения не соответствует максимальной передаче энергии от света к поверхностным волнам.

Следствием поверхностного плазмонного резонанса также является резонансное усиление электрического поля на поверхности металла. Так как связь молекул красителя с поверхностными плазмон-поляритонами осуществляется за счет эванесцентного поля $\left(E_{z}\right.$-проекция, перпендикулярная границе раздела), проникающего в среду с люминофором, то имеет смысл рассмотреть поведение этой величины. На рис. 4 представлена теоретически установленная зависимость квадрата модуля поля $\left|E_{z}\right|^{2}(\theta)$ (кривая 2) для точки в середине слоя PVA (расчет проведен по (9)). Видно, что данные эксперимента хорошо согласуются именно с поведением расчетной величины $\left|E_{z}\right|^{2}$, а не $R$ и, следовательно, можно сделать вывод, что величина $\left|E_{z}\right|^{2}$ является более корректной характеристикой для оценки свойств поверхностного плазмонно-связанного излучения образцов. Отметим также следующее обстоятельство: скорость спонтанного излучения молекулы или мощность излучения диполя прямо пропорциональны локальной плотности оптических состояний в точке нахождения излучателя. В этом смысле формула (3) описывает эквивалент локальной плотности. С другой стороны, для нахождения локальной плотности оптических состояний можно посчитать интенсивность поля в точке расположения диполя для волны, падающей на образец извне, при этом нужно учитывать соответствие между поляризацией волны и ориентацией диполя. По этой причине угловая зависимость $P$-поляризованной флуоресценции (на рис. 2 диаграмма направленности такой флуоресценции представлена кривой 1 ) ведет себя похожим образом с $\left|E_{z}\right|^{2}(\theta)$ и полностью совпадает с интенсивностью $P$-поляризованной волны, включающей также $x$-составляющую, т.е. с $\left|E_{x}\right|^{2}+\left|E_{z}\right|^{2}$ в месте нахождения диполя (при нормировке всех величин на максимум).

\section{Заключение}

Таким образом, были теоретически рассчитаны отражение образца, усиление поля, а также пространственное распределение связанной люминесценции для никелевой пленки в схеме Кречмана. Экспериментально измеренная плазмонно-связанная люминесценция Р6Ж демонстрирует узконаправленный профиль и значительную поляризацию и хорошо согласуется с расчетными данными. Оценка эффективности связи Р6Ж для никеля дает значение $45.6 \%$, что превышает значение, полученное для тонких пленок серебра $-30 \%$ [12]. Обнаружено, что минимумы диаграммы направленности коэффициентов отражения, рассчитанные методом матриц переноса, не совпадают с углами наибольшей интенсивности флуоресценции Р6Ж. При этом теоретически вычисленное усиление поля показало хорошее согласие с экспериментальными данными и может быть используемо вместо анализа коэффициента $R$ для прогнозирования свойств поверхностного плазмонно-связанного излучения образцов.

\section{Конфликт интересов}

Авторы заявляют, что у них нет конфликта интересов.

\section{Список литературы}

[1] Князев К.И., Якуненков Р.Е., Зулина Н.А., Фокина М.И., Набиуллина Р.Д., Торопов Н.А. // Опт. и спектр. 2018. T. 125. B. 4. C. 556. doi 10.21883/OS.2018.10.46711.187-18. Kniazev K.I., Yakunenkov R.E., Zulina N.A., Fokina M.I., Nabiullina R.D., Toropov N.A. // Opt. Spectr. 2018. V. 125. N 4. P. 578. doi 10.1134/S0030400X18100090 
[2] Jun Sun, Ziyang Li, Yinghui Sun, Liubiao Zhong, Jing Huang, Junchang Zhang, Zhiqiang Liang, Jianmei Chen, aLin Jiang // Nano Res. 2018. V. 11. N 2. P. 953. doi org/10.1007/s12274-017-1708-y

[3] Тихомирова Н.С., Самусев И.Г., Слежскин В.А., Зюбин А.Ю., Брюханов В.В. // ЖПС. 2017. Т. 74. С. 240. Tikhomirova N.S., Samusev I.G., Slezhkin V.A., Zyubin A.Y., Bryukhanov V.V. // J. Appl. Spectr. 2017. V. 84. N 2. P. 261. doi 10.1007/s10812-017-0461-z

[4] Acuna G.P., Moller F.M., Holzmeister P., Beater S., Lalkens B., Tinnefeld P. // Science. 2012. V. 338. N 6106. P. 506. doi 10.1126/science. 1228638

[5] Jun Dong, Zheng long Zhang, Hairong Zheng, Mentao Sun // Nanophotonics. 2015. V. 4. P. 472. doi 10.1515/nanoph2015-0028

[6] Nhu Hoa Thi Tran, Kieu The Loan Trinh, Jun-Ho Lee, Won Jung Yoon, Heongkyu Ju // Small. 2018. V. 14. P. 1801385. doi org/10.1002/smll.201801385

[7] Della Ventura B., Gelzo M., Battista E., Alabastri A., Schirato A., Castaldo G., Corso G., Gentile F., Velotta R. // ACS Appl. Mater. Interfaces. 2019. V. 11. N 4. P. 3753. doi 10.1021/acsami.8b20501

[8] Kum-Song Ho, Song-Jin Im, Ji-Song Pae, Chol-Song Ri, Yong-Ha Han, Joachim Herrmann // Sci. Rep. 2018. V. 8. P. 10584. doi 10.1038/s41598-018-28567-8

[9] Kum-Song Ho1, Song-Jin Im1, Ji-Song Pae1, Chol-Song Ri1, Yong-Ha Han1, Joachim Herrmann2 // Adv. Opt. Mater. 2013. V. 1. P. 10. doi 10.1038/s41598-018-28567-8

[10] Calander N. // J. Phys. Chem. B. 2005. V. 109. P. 13957. doi $10.1021 / \mathrm{jp} 0510544$

[11] Hansen W.N. // J. Opt. Soc. Am. 1968. V. 58. N 3. P. 380. https://doi.org/10.1364/JOSA.58.000380

[12] Gryczynski I., Malicka J., Gryczynski Z., Lakowicz J.R. // Anal. Biochem. 2004. V. 324. P. 170.

doi 10.1016/j.ab.2003.09.036 